\title{
Classification of Birds and UAVs based on Radar Polarimetry
}

\author{
Børge Torvik, Member, IEEE, , Karl Erik Olsen and Hugh Griffiths, Fellow, IEEE
}

\begin{abstract}
This letter aims to show the potential of using polarimetric parameters to distinguish between large birds and unmanned aerial vehicles (UAV) of comparable size in the context of a modern long range air defence radar. Time is a critical resource in such systems and techniques for robust noncooperative target recognition (NCTR) not relying on spatial resolution or long dwell times are highly desired. Furthermore, methods less dependent on target micro-motion are in many cases required. Methods exploiting polarimetric features are shown to have potential in both cases. An experiment in S-band shows that a simple Nearest-neighbor classifier can achieve good separation between UAVs and birds both with and without detectable micromotion based on a set of polarimetric parameters alone.
\end{abstract}

Index Terms-Radar, Radar target classification, Radar signature, Radar polarimetry, Radar measurements

\section{INTRODUCTION}

$\mathbf{L}$ OW price, high availability and low operational complexity have the last years contributed to considerable use of small UAVs or drones for a wide range of applications. Practically controllable by anyone, these may cause problems to both civilian and military authorities responsible for air safety and national security. The demand for reliable detection and classification of small airborne targets is rapidly emerging.

Long range air defence radars are key sensors for surveillance of airborne platforms in vast volumes. However, low altitude, velocity and radar cross section (RCS) are factors complicating the detection of small targets compared to conventional manned aircraft. Low frequency, limited bandwidth and short dwell times traditionally found in such systems have confined their ability in NCTR. Nevertheless, modern radars of the class are sensitive sensors developed for detection of physically small or potentially signature reduced targets at considerable distances.

The demand for detection of low signature and slow moving targets potentially introduce unwanted bird detections previously largely avoided by sensitivity time control (STC) and wide clutter rejection filters. The similarity in RCS and motion pattern between small UAVs and birds are key challenges associated with separation between the classes. In [1] Moon discussed the suitability for track based classification and concluded that there is considerable overlap between bird tracks and those of man-made targets. Although track and context based information may give important input to classification, the need for other discriminants seems unavoidable.

The problem of differentiation between birds and small unmanned targets by radar is relatively new in the literature. However, radar observations of birds have been reported in the fields of ornithology [2], radar meteorology and aviation security for decades. An overview of research up to 1985 is found in [3]. The proposed techniques can be divided roughly into three categories depending on the target scattering properties being exploited. These are target RCS modulation, micro-Doppler and polarization techniques. RCS modulation originating from repetitive wing beat pattern or rotor blade flashes as well as micro-Doppler shifts from the same scatterers are believed to be robust features for class separation as long as their RCS is large enough. Observations of these two effects in bird data were first described in [4] and [5] respectively. More recent reports on RCS modulations for small target classification in X-band can be found in [6], [7], and on micro-Doppler signatures in [8]-[11].

The major part of publications discussing utilization of polarimetric scattering effects for classification of small airborne targets originates from the meteorological radar community. Polarimetric features have been used to distinguish between different kinds of precipitation, but also for separation between precipitation, insects and birds [12]-[16]. In remote sensing different target decomposition techniques are proposed for classification of the surface in synthetic aperture radar (SAR) images. A review of such techniques is found in [17].

This letter addresses the problem of differentiating between birds and small UAVs in modern long range defence radar. Time is a critical resource in these systems and focus has been on finding time efficient methods. The main idea behind the presented work is that polarimetric parameters contain useful information about small targets, and that this is well preserved when bandwidth, dwell time and carrier frequency are reduced. This in contrast to classic NCTR methods relying on spatial or velocity resolution.

In some cases traditional single polarization data are sufficient for classification, even for small targets. This is discussed in Section IV. However, more information can often be extracted from polarimetric data. The same section presents spectrograms of selected polarimetric variables from data of a flapping bird and how these may contribute to identification of the underlying scattering mechanisms. The letter is rounded off with a review of an experiment investigating classification in S-band based on polarimetric parameters alone. Special attention is given to the challenging problem of separating between soaring birds and small drones with plastic rotor blades, which are classes characterized by insignificant microDoppler spectrum and non-periodic RCS modulations.

\section{SCATTERING FROM SMALL TARGETS}

Target signatures are inherent in echo amplitudes and phases dependent on the scatterer's physical size, shape, dielectric 
TABLE I

POLARIMETRIC PARAMETERS USED FOR CLASSIFICATION

\begin{tabular}{|c|c|c|}
\hline Parameter & Relation to $\mathrm{S}$ & Explanation \\
\hline$\delta$ & $2\left\langle\left|S_{h v}\right|^{2}\right\rangle /\left\langle\left|S_{h h}\right|^{2}\right\rangle$ & $\begin{array}{l}\text { Linear depolarization } \\
\text { ratio }\end{array}$ \\
\hline$\gamma$ & $\left\langle\left|S_{v v}\right|^{2}\right\rangle /\left\langle\left|S_{h h}\right|^{2}\right\rangle$ & $\begin{array}{l}\text { Differential polariza- } \\
\text { tion ratio }\end{array}$ \\
\hline$\rho$ & $\left\langle S_{h h} S_{v v}^{*}\right\rangle / \sqrt{\left(\left\langle\left|S_{h h}\right|^{2}\right\rangle\left\langle\left|S_{v v}\right|^{2}\right\rangle\right.}$ & $\begin{array}{l}\text { Co-polarized correla- } \\
\text { tion coefficient }\end{array}$ \\
\hline$\beta$ & $\left\langle S_{h h} S_{h v}^{*}\right\rangle / \sqrt{\left\langle\left|S_{h h}\right|^{2}\right\rangle\left\langle\left|S_{h v}\right|^{2}\right\rangle}$ & $\begin{array}{l}\text { Cross-polarized cor- } \\
\text { relation coefficient }\end{array}$ \\
\hline$\epsilon$ & $\left\langle S_{h v} S_{v v}^{*}\right\rangle / \sqrt{\left\langle\left|S_{h v}\right|^{2}\right\rangle\left\langle\left|S_{v v}\right|^{2}\right\rangle}$ & $\begin{array}{l}\text { Cross-polarized cor- } \\
\text { relation coefficient }\end{array}$ \\
\hline$H$ & See [19] & Entropy \\
\hline$A$ & See [18] & Anisotropy \\
\hline $\bar{\alpha}$ & See [19] & $\begin{array}{l}\text { Polarimetric } \\
\text { eigenvector parameter }\end{array}$ \\
\hline $\bar{\beta}$ & See [19] & $\begin{array}{l}\text { Orientation angle es- } \\
\text { timate }\end{array}$ \\
\hline
\end{tabular}

properties as well as the wavelength and orientation relative to the illuminating electrical field. In the monostatic case we assume $S_{h v}=S_{v h}$ and the backscattered field is described by the scattering matrix $\mathbf{S}$. On a horizontal-vertical basis this can according to [18] be expressed as:

$$
\mathbf{S}=\left[\begin{array}{ll}
\left|S_{h h}\right| e^{j \phi_{h h}} & \left|S_{h v}\right| e^{j \phi_{h v}} \\
\left|S_{v h}\right| e^{j \phi_{v h}} & \left|S_{v v}\right| e^{j \phi_{v v}}
\end{array}\right]
$$

The goal is to investigate if observations of $\mathbf{S}$ can reveal information useful for classification of small targets. Although these are both physically and electrically small there may exist several scattering mechanisms contributing to the total scattered field, each with individual polarimetric signatures.

Several polarimetric parameters extractable from $\mathbf{S}$ are candidates as features for classification. The ones used in this study are found in Table I. The first group of variables is the so-called polarimetric intercorrelation parameters $(\delta-\epsilon)$ [18]. These are formed from average values of elements of $\mathbf{S}$ over a time interval $\tau_{\text {avg }}$.

The last four parameters $(H-\bar{\beta})$ are outputs from a polarimetric target decomposition technique with the benefit of being independent of polarimetric basis. Such methods are commonly used in remote sensing for classification of the ground surface in SAR images. Here the dominating scattering effect is expressed as a sum of independent elements, which in turn are associated with physical scattering mechanisms. For the experiments discussed in this paper the $\mathrm{H} / \mathrm{A} / \bar{\alpha}$ method suggested by Cloude and Pottier [19] was selected. Due to required speckle filtering of SAR images the physical scattering mechanism is not interpreted from the scattering matrix directly, but on the basis of an averaged coherency matrix $\langle T\rangle$. We apply this method normally used in imaging radar on one dimensional radar data. Although not being equally susceptible to speckle in the small target classification case, the backscattered signal is by no means stationary and the method is applied directly with the ensemble average replaced with a time average over an interval $\tau_{\text {avg }}$.
TABLE II

TARGET CLASSES

\begin{tabular}{lll}
\hline Class & Description & Target type \\
A & UAV with carbon fiber rotor blades & DJI Phantom II \\
B & Bird with flapping wings & Large sea birds \\
C & UAV with plastic rotor blades & 3D solo \\
D & Gliding birds & Large sea birds \\
\hline \hline
\end{tabular}

\section{Measurements}

Large wild sea birds and two types of small drones were measured with the BirdRAD system in S-band. This is a ground based experimental quadrature polarization radar designed to investigate the scattering properties of small targets in both L- and S-band simultaneously. The measurements covered in this letter were collected at $3.25 \mathrm{GHz}$ with a pulse repetition frequency (PRF) of $10 \mathrm{kHz}$. The resolution in range was $3 \mathrm{~m}$ and $8^{\circ}$ in both azimuth- and elevation-angle. Single targets were manually tracked in angle by use of a video camera aligned with the antenna and isolated by signal processing in range and velocity for further micro-Doppler and polarimetric analysis.

The targets covered are found in Table II. The birds, mainly sea eagles and black-backed gulls, were measured both soaring and flapping. The observations were done from a wide range of aspect angles and at distances between 0.3-1.5 km. The UAVs, a DJI Phantom II [20] with carbon rotor blades and a 3D solo [21] with plastic blades were measured performing race track maneuvers at a range of $0.3-0.4 \mathrm{~km}$.

This experiment was limited in that only two small UAVs and only large birds are included. Nevertheless, a wide variation of aspect angles and target behavior was covered in all classes. Other potential weaknesses which could lead to optimistic results may be connected to the birds being measured at many distances, whereas data of the UAVs were collected at ranges from $0.3-0.4 \mathrm{~km}$ only. This is still not believed to have influenced the results significantly in any way.

\section{RESUlTS AND DISCUSSION}

In some cases satisfactory classification of small targets can be expected based on single polarization data alone. In other circumstances polarimetric parameters can improve classification, whereas for some targets such a description is believed to be required in order to distinguish between target classes. All three situations are discussed in the following paragraphs.

\section{A. Single polarization classification}

As mentioned, dielectric properties of certain target parts are important. Whereas plastic rotors tend to be virtually invisible to radar, carbon fiber blades of the same size and shape have significantly larger RCS [22]. Rotor blades and bird wings tend to be electrically small in long range radar frequency bands and are assumed to be well modeled as dipole scatterers. According to [18] the monostatic scattering matrix for a dipole 


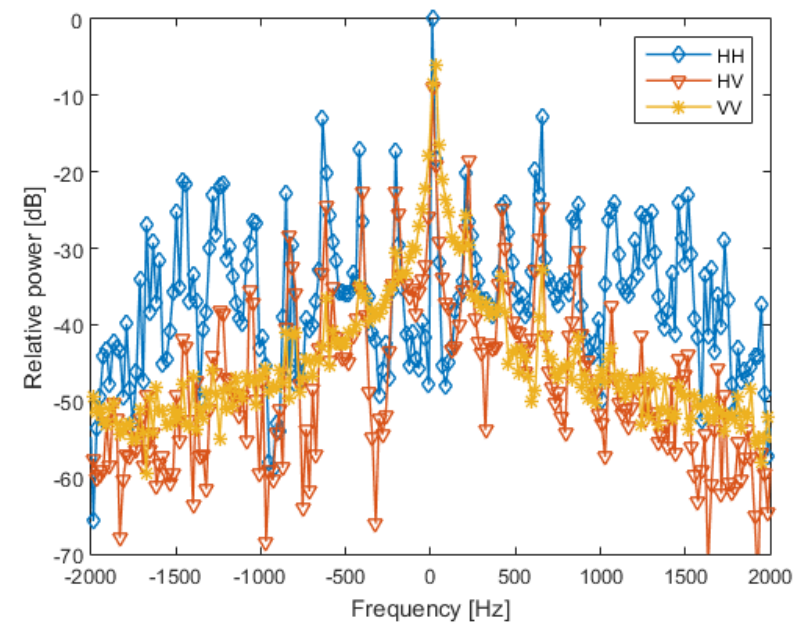

Fig. 1. Periodogram of backscattered power from DJI. $\tau=1 \mathrm{~s}$

is dependent on its orientation $\theta$ relative to the illuminating E-field about the radar line of sight as:

$$
\mathbf{S}_{\text {dipole }}=\left[\begin{array}{cc}
\cos ^{2}(\theta) & \frac{1}{2} \sin (2 \theta) \\
\frac{1}{2} \sin (2 \theta) & \sin ^{2}(\theta)
\end{array}\right]
$$

Based on these assumptions, Equation (2) suggests that $\mathrm{HH}-$ polarization is the best choice of polarization when the blade rotation is in the horizontal plane. The periodogram of echoes from the DJI drone with carbon fiber rotor blades in Fig. 1 confirms this. Here the translational velocity of the drone is compensated for and the target body response is centered at $0 \mathrm{~Hz}$. The harmonics present in the HH-channel and significantly attenuated in the HV-channel are results of many periodic blade flash events during the processing interval of one second. In contrast the VV-channel has no clear trace of such flashes. This example gives a first glimpse of how important polarization can be for classification.

Rotating carbon fiber blades and propellers are examples of target parts constituting distinct signatures both in time and frequency domain. In terms of RCS modulation effects, cepstrum analysis is an effective tool in detecting the presence of returns from such by utilizing harmonics in the data. Although less efficient, the same method can be used to estimate the wing beat frequency of flapping bird wings. In this case variation with aspect angle tends to be larger, the periodicity somewhat less and the required observation time must be increased radically due to the long wing beat period.

Micro-Doppler signatures of targets in class A and B contain information in the form of phase modulations from rotating target parts. In order to utilize this for classification such scatterers have to have sufficiently large RCS and relative velocity in order to be resolved from the often much larger target body. For a given target behavior and carrier frequency this resolvability can be influenced by selection of the length of the coherent processing interval $\tau$ and the shape of the window function selected for spectral estimation.

As long as the rotating parts of targets in class A and $\mathrm{B}$ can be resolved in velocity, micro-Doppler signatures are generally prominent, recognizable and distinguishable between

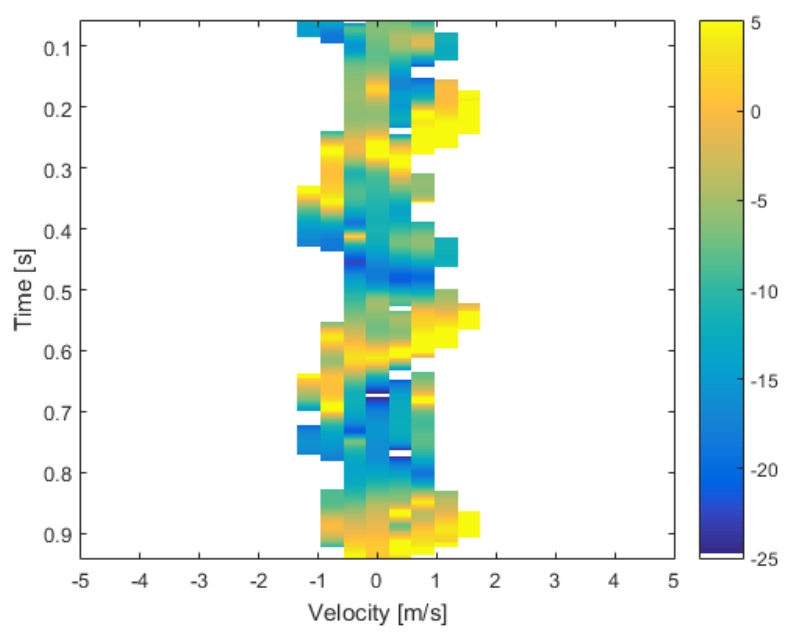

Fig. 2. Linear depolarization ratio $\delta$ of flapping black-backed gull in S-band. $\tau=120 \mathrm{~ms}$

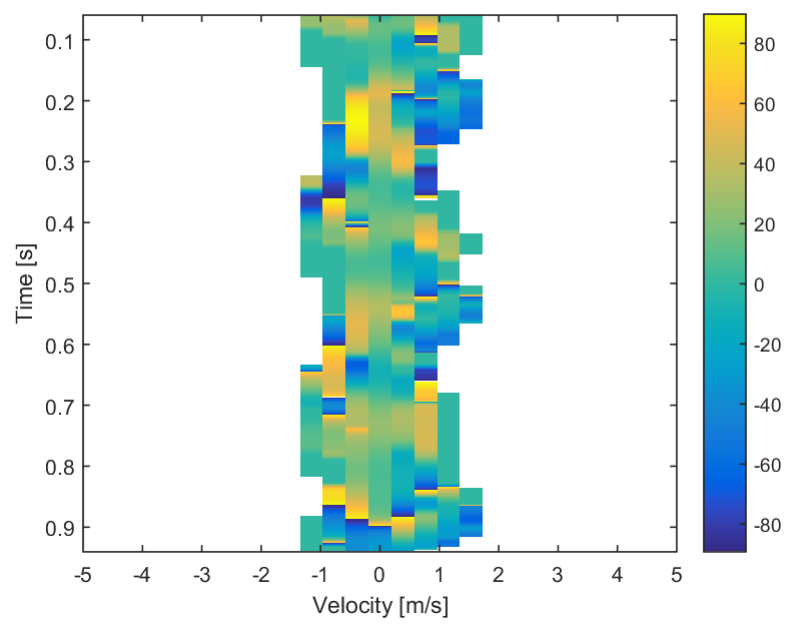

Fig. 3. $\bar{\beta}$ used as estimate for bird body and wing orientation around the radar line of sight. Flapping black-backed gull in S-band. $\tau=120 \mathrm{~ms}$

the classes. However, there is reason to believe that additional information can be extracted from polarimetric data that can contribute to classification at a more detailed level.

\section{B. Polarimetric signatures of rotating scatterers}

Extraction of polarimetric parameters can either be done in time domain or in time-frequency domain. The latter give the advantage of potentially resolving scatterers rotating around reflectors constituting the target body, and thereby isolate their polarimetric signature. This is beneficial when the RCSs of the rotating parts are small compared to that of the main body and their contribution to the time-domain signature are easily drowned.

In the small airborne target classification problem, polarimetric data may provide information both on the scattering mechanisms as well as the orientation of resolved parts. All variables in Table I were analyzed in the time-frequency domain the same way as shown for the linear depolarization 
ratio $\delta$ in Fig. 2 and an orientation angle estimate $\bar{\beta}$ in Fig. 3 . The translational velocity of the bird is in both figures compensated for, and the rotational movement of the bird wings can be seen in intervals where these are resolvable from bird body. High values of $\delta$ in the first figure indicate areas where cross-polarization ratio is high and is seen to form a pattern with the wing beat frequency of $3-4 \mathrm{~Hz}$ observed in video. In the latter figure, the value $\bar{\beta}$ from the $H / A / \bar{\alpha}$ method indicates the preferred orientation of the scatterer around the radar line of sight. Interesting signatures are found for many of the other polarimetric variables as well and together these are believed to provide valuable information allowing for more detailed classification as well as target behavior analysis. However, in the remainder of this paper we focus on the more challenging classification problems involving short dwell times and targets with no detectable rotating parts.

\section{Classification based on polarimetric data alone}

Gliding birds and UAVs with plastic rotors are classes characterized by insignificant micro-Doppler shifts and lack of interpretable periodic RCS modulations, even for long $\tau$. Separation between such classes with similar RCS and movement pattern is for these reasons difficult by traditional NCTR techniques utilizing one single polarization alone.

The polarimetric parameters in Table I extracted from timedomain data were fed to a simple Nearest-neighbor classifier in two different tests. The first one with data from classes $\mathrm{C}$ and D only, for evaluation of the separability of classes with no detectable micro-motion. The second test included targets from all four classes, however, the classifier was trained to separate between birds and man-made targets only. A five fold cross validation was applied in the process of estimating the accuracy of the classifier in both cases. This means that the data were randomly divided into five equally large portions, and one subset at a time was incrementally used to validate the model trained by the remaining four data subsets. Different values of $\tau_{\text {avg }}$ were tested and a sequential feature selection method was used to evaluate the contribution of each parameter to the overall classification. The evaluation was not done for all $2^{N}$ combinations of the $N$ features. Instead, the performance was estimated by sequentially adding the best feature until the improvement of misclassification rate was insignificant. This means that for each round of evaluation the feature providing the best improvement was identified and excluded from the next round of evaluation. The fitness measure used for ranking the parameters was the misclassification rate here defined as the portion of all measurements wrongly classified.

Figure 4 shows the result of this ranking of parameters when the classifier was trained to separate between class $C$ and D only. The evaluation of parameters were done for $\tau_{\text {avg }}$ ranging from 2 to $150 \mathrm{~ms}$. The parameters are seen to contribute differently for different averaging times. For small values the anisotropy A is good feature for separation between the classes and a misclassification rate of $5.4 \%$ is reached by classification on this parameter alone. For larger values of $\tau_{\text {avg }}$, A is a much poorer discriminant. However, now other parameters contribute more. For $\tau_{\text {avg }}=20$ and $150 \mathrm{~ms}, \bar{\alpha}$ is

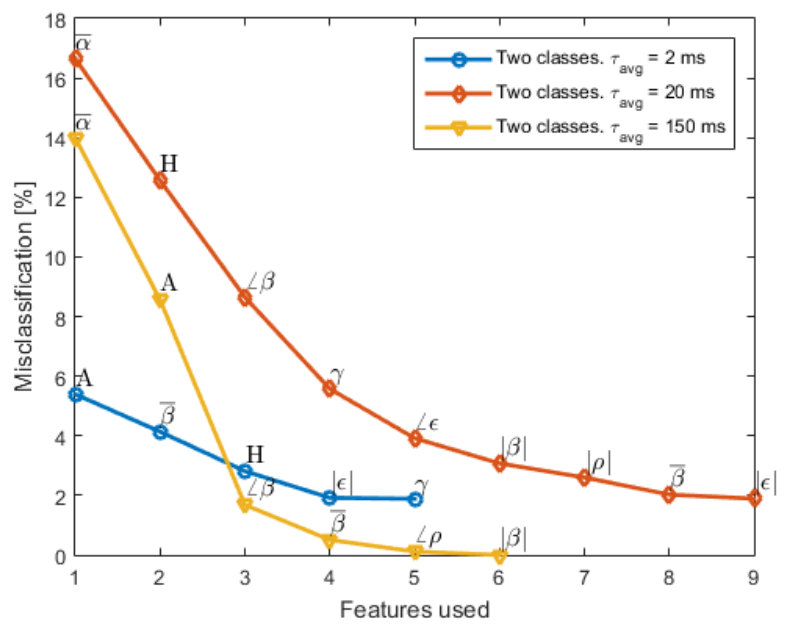

Fig. 4. Results of automatic feature selection for separation of soaring birds and $3 \mathrm{D}$ solo drone with undetectable plastic rotor blades

the best discriminant. This parameter holds information on odd or even bounce scattering effects. This can be interpreted as the classes are dominated by different scattering mechanisms. Interestingly, there is a range of values of $\tau_{\text {avg }}$ where $\mathrm{A}$ has lost much its discriminating power and four more features are need to reach comparable misclassification level, see red line with $\tau_{\text {avg }}=20 \mathrm{~ms}$. However, for $\tau_{\text {avg }}=150 \mathrm{~ms}$ the other parameters contribute more and the correct classification level reaches $100 \%$ by use of 6 parameters. It is worth emphasizing that the $\tau_{\text {avg }}$ was chosen to both define the averaging time for both $\mathrm{H}, \mathrm{A}, \bar{\alpha}, \bar{\beta}$ and the intercorrelation parameters. Individually customization of $\tau_{\text {avg }}$ may improve classification.

Inspired by these first encouraging results, classes $\mathrm{A}$ and B were included in a similar test. However, the classifier was now to distinguish between UAV and bird only. It is worth emphasizing that better separation between the two classes can be expected by inclusion of non-polarimetric variables, at least for long $\tau_{\text {avg }}$. However, the research question of this test was to investigate how the set of polarimetric parameters performed when dwell time was limited. The results presented in Fig. 5 are rather similar to those found in the two class problem. The classification score is acceptable even for short values of $\tau_{\text {avg }}$.

The confusion matrix in Table III shows the classification score in number of observations and percentage per true class for the classifier trained for separation of classes A-D and for $\tau_{\text {avg }}=150 \mathrm{~ms}$. Values close to $100 \%$ on the diagonal indicate that the selected polarimetric time domain parameters contain information useful for separation between all four classes.

\section{CONCLUSION}

This letter has shown that polarimetric data may contribute to separation between small unmanned aerial targets and birds in the context of modern long range air defence radar. The fundamental assumption, that polarimetric information is well preserved as carrier frequency, bandwidth and dwell time is reduced, is supported by experimental results. This may lead to new NCTR techniques capable of distinguishing between 


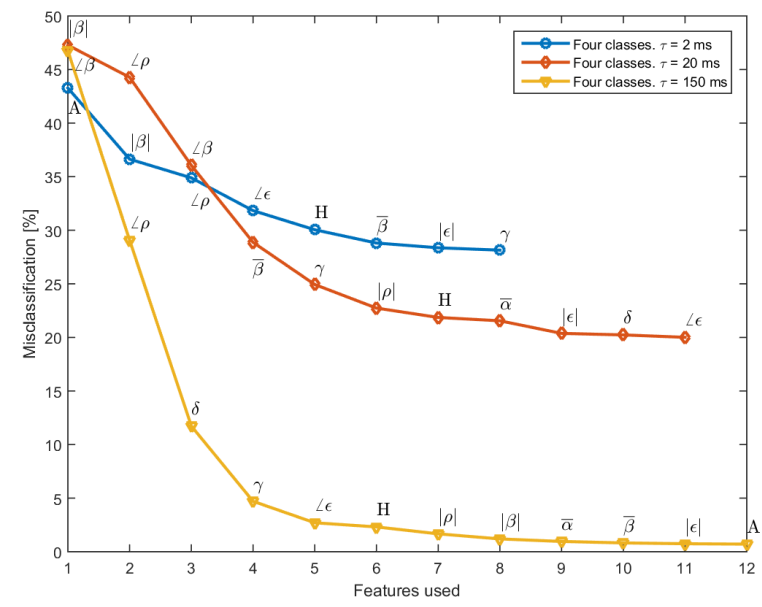

Fig. 5. Automatically selected features for separation between birds and UAVs among four targets: gliding bird, flapping bird, 3D solo with plastic rotor blades and DJI Phantom II with carbon fiber rotor blades

TABLE III

CONFUSION MATRIX FOR FOUR CLASS PROBLEM. $\tau_{a v g}=150 \mathrm{~ms}$

\begin{tabular}{|c|c|c|c|c|}
\hline & \multicolumn{4}{|c|}{ Predicted class } \\
Class & $\mathrm{A}$ & $\mathrm{B}$ & $\mathrm{C}$ & $\mathrm{D}$ \\
\hline $\mathrm{A}$ & $335 / 100 \%$ & $0 / 0 \%$ & $0 / 0 \%$ & $0 / 0 \%$ \\
\hline $\mathrm{B}$ & $6 / 0.1 \%$ & $5078 / 98.8 \%$ & $4 / 0.1 \%$ & $51 / 1.0 \%$ \\
\hline $\mathrm{C}$ & $0 / 0 \%$ & $3 / 0.8 \%$ & $383 / 99.2 \%$ & $0 / 0.0 \%$ \\
\hline $\mathrm{D}$ & $1 / 0.0 \%$ & $23 / 1.1 \%$ & $0 / 0 \%$ & $2147 / 98.9 \%$ \\
\hline
\end{tabular}

small man-made targets and birds without relying on spatial resolution or long dwell times for velocity resolution. Even in cases where targets are not associated with any detectable micro-motion, separation between the classes is shown to be possible with promising classification levels.

Analysis of a set of polarimetric parameters was done in the time-frequency domain, such that scatterers with different micro-motion were resolved in time and velocity. This enables the possibility of investigating the polarimetric signature and orientation of scatterers often dominated by others in the time domain. Many of these give valuable information that may be used for more detailed target classification and motion analysis. Despite these scatterers are often drowned by target body reflections, classification results in time domain are promising. The physical mechanisms forming the foundation for classification are not investigated in detail, however, there is reason to believe that dominating scattering mechanisms of birds and UAVs are somewhat different. The fact that the observed targets and its parts are found in the resonance and Rayleigh scattering regions, leads to the idea of a polarimetric signature dominated by the overall target shape. In this context the signature of a roughly ellipsoidal shaped bird with dipole like wings is believed to differ from the more random scattering mechanisms originating mainly from the inside of UAVs. The ranking of polarimetric parameters presented can shed light on which physical mechanisms contribute to classification. Indications of systematically different values of $\bar{\alpha}$ and the co-polarized phase difference $\phi_{v v}-\phi_{h h}$ is found, which is believed to be caused by less multi bounce scattering and more creeping wave effects observed in bird data respectively.

The presented experiment has provided a first proof-ofconcept and forms a valuable basis for further work. Future research will include more targets in all classes, investigation of more polarimetric parameters as well as increased focus on understanding the physical scattering mechanisms separating the classes.

\section{REFERENCES}

[1] J. R. Moon, "Effects of birds on radar tracking systems," in RADAR 2002, Edinburgh, 2002, pp. 300-304.

[2] E. Eastwood, Radar Ornithology. Methuen \& Co, Ltd., 1967.

[3] C. R. Vaughn, "Birds and insects as radar targets: A review," Proceedings of the IEEE, vol. 73, no. 2, pp. 205-227, Feb 1985.

[4] A. H. LaGrone, A. P. Deam, and G. B. Walker, "Angels, insects and weather," Journal of research, Radio science, vol. 68D, pp. 865-901, 1964.

[5] L. C. Ireland and T. C. Williams, "Radar observations of bird migration over Bermuda," in A conference on the biological aspects of the bird/aircraft collision problem, S. G. Jr., Ed., Clemson, South Carolina, May 1974, pp. 383-408.

[6] B. Torvik, A. Knapskog, O. Lie-Svendsen, K. E. Olsen, and H. D. Griffiths, "Amplitude modulation on echoes from large birds," in Proceedings of the 11th European Radar Conference (EuRAD), 2014, Rome, Italy, Oct 2014, pp. 177-180.

[7] R. I. A. Harmanny, J. J. M. de Wit, and G. P. Cabic, "Radar microdoppler feature extraction using the spectrogram and the cepstrogram," in Proceedings of the 11th European Radar Conference (EuRAD), Rome, 2014, pp. 165-168.

[8] C. M. Alabaster, E. J. Hughes, and D. W. Forman, "Is it a bird or is it a plane?" in Proc. IEEE 6th International Conference on Waveform Diversity and Design, Lihue, HI, 2012.

[9] B. Torvik, K. E. Olsen, and H. D. Griffiths, "K-band radar signature analysis of a flying mallard duck," in Proc. 14 th International Radar Symposium (IRS), 2013, vol. 2, Dresden, Germany, 2013, pp. 584-591.

[10] - "X-band measurements of radar signatures of large sea birds," in International Radar Conference, 2014, Lille, France, Oct 2014, pp. 1-6.

[11] J. J. M. de Wit, R. I. A. Harmanny, and G. Prmel-Cabic, "Micro-doppler analysis of small UAVs," in 9th European Radar Conference (EuRAD), 2012, Rome, Italy, 2012, pp. 210-213.

[12] J. W. Wilson, T. M. Weckwerth, J. Vivekanandan, R. M. Wakimoto, and R. W. Russell, "Boundary layer clear-air radar echoes: Origin of echoes and accuracy of derived winds," Journal of Atmospheric and Oceanic Technology, vol. 11, no. 5, pp. 1184-1206, 1994.

[13] D. S. Zrnic and A. V. Ryzhkov, "Polarimetry for weather surveillance radars," Bulletin of the American Meteorological Society, vol. 80, no. 3, pp. 389-406, 1999.

[14] V. M. Melnikov, R. R. Lee, and N. J. Langlieb, "Resonance effects within s-band in echoes from birds," Geoscience and Remote Sensing Letters, IEEE, vol. 9, no. 3, pp. 413-416, 2012.

[15] P. Zhang, A. V. Ryzhkov, and D. S. Zrnic, "Detection of birds and insects using polarimetric radar observation," in Preprints, 11th Conference on Aviation, Range, and Aerospace Meteorology, vol. 5, Hyannis, MA, 2004. [Online]. Available: https://ams.confex.com/ams/11aram22sls/techprogram/paper_81616.htm

[16] D. S. Zrnic and A. V. Ryzhkov, "Observations of insects and birds with a polarimetric radar," IEEE Transactions on Geoscience and Remote Sensing, vol. 36, no. 2, pp. 661-668, 1998.

[17] S. R. Cloude and E. Pottier, "A review of target decomposition theorems in radar polarimetry," IEEE Transactions on Geoscience and Remote Sensing, vol. 34, no. 2, pp. 498-518, Mar 1996.

[18] J. S. Lee and E. Pottier, Polarimetric Radar Imaging: From Basics to Applications. CRC Press, 2009.

[19] S. R. Cloude and E. Pottier, "An entropy based classification scheme for land applications of polarimetric sar," IEEE Transactions on Geoscience and Remote Sensing, vol. 35, no. 1, pp. 68-78, Jan 1997.

[20] "http://www.dji.com/."

[21] "https://store.3dr.com/.",

[22] M. Ritchie, F. Fioranelli, H. D. Griffiths, and B. Torvik, "Micro-drone RCS analysis," in IEEE Radar Conference, Johannesburgh, South Africa, 2015, pp. 452-456. 